\title{
Relación Estado-sociedad civil en las políticas de desarrollo socio-productivo en Argentina contemporánea
}

\author{
Malena Victoria Hopp \\ Universidad de Buenos Aires (UBA)
}

\section{Relación Estado-sociedad civil en las políticas de desarrollo socio-productivo en Argentina contemporánea}

Resumen: El artículo reflexiona acerca de la relación entre las instituciones estatales y la sociedad civil en las políticas de desarrollo socio-productivo, implementadas en la Argentina a partir del año 2003. Para ello se indaga cuál es el rol asignado a la participación de los destinatarios y las organizaciones de la sociedad civil en estas políticas y los supuestos acerca de las formas de participación que subyacen en sus normativas. Luego, se analiza cómo se desarrolla efectivamente esta articulación entre Estado y sociedad civil en el proceso de implementación de los programas y las dificultades que se presentan, a partir del caso de una cooperativa de trabajo que fue apoyada por estos programas. En cuanto a la metodología se utilizó un enfoque plural que articuló el análisis documental con el uso de técnicas cualitativas de corte etnográfico.

Palabras clave: Políticas sociales. Economía social. Estado. Sociedad civil.

\section{Relação estado-sociedade civil nas políticas de desenvolvimento socioprodutivas na Argentina contemporânea}

Resumo: O Artigo reflete sobre a relação entre as instituições estaduais e a sociedade civil nas políticas de desenvolvimento socioprodutivas, implementadas na Argentina a partir de 2003. Para tanto, examina-se a função atribuída à participação dos beneficiários e as organizações da sociedade civil nessas políticas e os supostos acerca das formas de participação que subjazem em suas normativas. Em seguida, analisa-se o desenvolvimento efetivo dessa articulação entre estado e sociedade civil, no processo de execução dos programas e as dificuldades que surgem, a partir do caso de uma cooperativa de trabalho que foi apoiada por tais programas. Em relação à metodologia, foi usada uma abordagem plural que articulou a análise documentária junto com técnicas qualitativas de tipo etnográficas.

Palavras-chave: Políticas sociais. Economia social. Estado. Sociedade civil.

The Relationship between the State and Civil Society in Socio-Productive Development in Argentina Today

Abstract: This article reflects on the relationship between government institutions and civil society in socio-productive development policies implemented in Argentina since 2003. It examines the role assigned to the participation of civil society beneficiaries and organizations in these policies and the suppositions about the forms of participation established in their norms. It then looks at the effective development of this articulation between the state and civil society in the process of execution of the programs and difficulties that arise at a labor cooperative that was supported by these programs. A plural methodological approach was used that articulated document analysis to qualitative ethnographic techniques.

Key words: Social policies. Social economy. State. Civil society. 


\section{Introducción}

En el año 2003, con la asunción de Néstor Kirchner como presidente y de Alicia Kirchner como Ministra de Desarrollo Social, el gobierno propuso una revisión de las políticas sociales que se estaban implementando desde la década del '90. Sus argumentos hacían referencia al modelo neoliberal y las medidas de ajuste estructural, como causas de los problemas sociales, al mismo tiempo que denunciaban la focalización y el asistencialismo de las políticas destinadas a atenderlos. En oposición a ese modelo, desde el discurso ${ }^{1}$ oficial se propuso una nueva orientación, según la cual "la mejor política social es la creación de empleos."

En este contexto de cambios en la gestión del gobierno y en la política social, comenzó a implementarse - desde el Ministerio de Desarrollo Social (MDS) - el Plan Nacional de Desarrollo Local y Economía Social "Manos a la Obra". A partir del cual, la economía social adquirió un rol más relevante como estrategia de "inclusión social". El objetivo de este Plan es lograr un desarrollo social económicamente sustentable que permita generar empleo, mejorar la calidad de vida de las familias y promover la integración de personas o grupos en situación de vulnerabilidad social y pobreza, a través de la generación de autoempleo y la participación en los espacios comunitarios.

Ese mismo año, y en consonancia con la nueva orientación propuesta, el Ministerio de Trabajo, Empleo y Seguridad Social (MTESS) hizo explícita la búsqueda de "políticas activas que [...] favorezcan la creación de empleo genuino y la transformación de programas asistenciales en políticas de integración social y productiva de la población desocupada"2, e impulsó la creación de diversos planes de autoempleo ${ }^{3}$.

Profundizando esta nueva línea de política social, en agosto del 2009, se lanzó el Programa Ingreso Social con Trabajo, "Argentina Trabaja”, cuyo objetivo es la "inclusión social a través del trabajo" y la promoción de la organización cooperativa (ARGENTINA, 2009). Éste se implementa en el marco del Plan Nacional de Desarrollo Local y Economía Social "Manos a la Obra" y propone la creación de 100 mil nuevos puestos de trabajo, en una primera etapa en la Provincia de Buenos Aires, que luego se ampliará y se extenderá a todo el territorio nacional.

Estos programas pueden ser definidos como políticas de desarrollo socio-productivas, entendidas como intervenciones sociales del Estado, que pueden considerarse como políticas sociales, porque su objeto es la reproducción de la vida de grupos sociales (DANANI, 1996), al mismo tiempo que como políticas económicas. Dado que intervienen en la distribución primaria del ingreso y la intervención en las condiciones de vida, se realiza a través de prestaciones típicamente económicas (como subsidios o créditos), que estimulan la producción para el mercado (ABRAMOVICH, 2007). En este sentido, estas nuevas políticas y las unidades productivas que emergen o son apoyadas por las mismas, tensionan la tradicional distinción entre la esfera de la política social y la política económica, al mismo tiempo que redefinen el rol del Estado y suponen la activa participación de la sociedad civil ${ }^{4}$ en el proceso de implementación de las mismas.

En este marco, el objetivo de este artículo es analizar las relaciones entre las instituciones estatales y la sociedad civil que promueven las políticas de desarrollo socio-productivo, implementadas en la Argentina desde el año 2003. Para ello, se indaga cual es el rol asignado a la participación de los destinatarios y las organizaciones de la sociedad civil, en estas políticas, y los supuestos acerca de estas formas de participación que subyacen en las normativas de los mencionados programas. Luego, se analiza como se desarrolla efectivamente la articulación entre el Estado y la sociedad civil en el proceso de implementación de los programas y las dificultades que allí se presentan, a partir de la experiencia de una cooperativa de trabajo que fue apoyada por estos programas.

\section{Metodología}

En esta investigación se utilizó un enfoque plural, que articuló técnicas cualitativas de corte etnográfico, entrevistas y observaciones con participación, con el análisis documental de las normativas de los programas, informes de gestión y evaluación de los mismos.

El referente empírico, seleccionado para realizar el trabajo de campo, fue una cooperativa de trabajo, localizada en la Ciudad de Buenos Aires, que fue apoyada por distintos planes de desarrollo socioproductivo. El mismo se desarrolló entre los meses de abril) de 2009 y marzo del 2010. Mediante el trabajo de campo etnográfico, documentamos las prácticas y experiencias cotidianas de los sujetos que integran esta cooperativa.

El ámbito de lo cotidiano es el campo privilegiado de este enfoque (GEERTZ, 1994), dado que es allí donde se da el proceso de reproducción del hombre concreto que permite, a su vez, la reproducción de la sociedad (HELLER, 2002). El acercamiento a esta experiencia permitió explorar los sentidos que los sujetos le atribuyen a sus prácticas y a la relación que establecen con el Estado, a través de las políticas de promoción del desarrollo socio-productivo. Siguiendo las vinculaciones de los sujetos y sus prácticas cotidianas, realizamos observaciones en distintas dependencias estatales, acompañando a los integrantes de la cooperativa a realizar trámites rela- 
tivos a la gestión de recursos estatales provenientes de los planes que son objeto de análisis.

La investigación realizada cumple con los principios legales y éticos establecidos para las investigaciones con seres humanos y respeta lo acordado en la Declaración de Helsinki. Para esta publicación se ha solicitado el consentimiento de los sujetos y se ha asegurado la confidencialidad de sus testimonios. Asimismo, se ha cambiado el nombre de la cooperativa y de sus integrantes, a fin de garantizar el anonimato de los informantes.

\section{La participación de la sociedad civil "en el papel"}

En este punto analizaremos el rol asignado a la participación de los destinatarios y organizaciones de la sociedad civil, en las normativas de los programas de desarrollo socio-productivo ${ }^{5}$ y el modo en que es concebido el sujeto de estas políticas sociales.

El diagnóstico oficial ${ }^{6}$ que dio origen al Plan "Manos a la Obra" comenzó señalando los problemas centrales de la Argentina en tres niveles: a nivel macro social, se observaban las consecuencias del modelo neoliberal en la desestructuración del sistema productivo y las transformaciones en las regulaciones del trabajo, las cuales habían generado desempleo, subempleo, flexibilización y precarización laboral. Esto tuvo como correlato un aumento de la pobreza y de la cantidad de personas en situación de vulnerabilidad social. Para afrontar estas dificultades, un sector de esta población comenzó a desarrollar estrategias de generación de ingresos a través de emprendimientos productivos de baja calidad. Debido a los lineamientos del modelo de desarrollo neoliberal, éstos presentaban dificultades en la comercialización de su producción y para su sostenimiento.

A nivel local, el diagnóstico planteaba la existencia de actores sociales que se encontraban desarticulados y debilitados, lo cual limitaba el potencial de generación de un desarrollo socialmente sustentable y con equidad. Por último, afirmaba que los emprendedores no contaban con la suficiente experiencia en la gestión de emprendimientos productivos, tenían problemas para planificar su actividad económica y dificultades de acceso al sistema financiero y bancario.

A partir de este diagnóstico, se propuso diseñar el Plan "Manos a la Obra" que integró diversos programas focalizados y existentes en el Ministerio de Desarrollo Social, desde la década de 1990. Según la resolución n. 1.375/2004 (ARGENTINA, 2004) de creación del Plan, su propósito es "constituirse como un sistema de apoyo a las iniciativas de desarrollo socioeconómico local, destinado particularmente a los sectores de bajos recursos". Para ello se plantean tres objetivos generales: 1) contribuir a la mejora del ingreso de la población en situación de vulnerabilidad social en todo el país; 2) promover la economía social mediante el apoyo técnico y financiero a emprendimientos productivos de inclusión social generados en el marco de procesos de desarrollo local y 3) fortalecer las organizaciones públicas y privadas, así como los espacios asociativos y redes, promoviendo la descentralización de los diversos actores sociales de cada localidad. Tal como expresa la citada resolución, los ejes de este Plan son la promoción del desarrollo local y la "inclusión social", a través de la generación de empleo y la participación en los espacios comunitarios.

Desde el inicio, su implementación presentó una serie de desafíos organizacionales y de gestión que implicaron, entre otros,

[...]el impulso de mecanismos de coordinación con otras instituciones; la profundización y/o redefinición de los espacios de participación y control social, conformando mesas de trabajo con redes de organizaciones de la sociedad civil y fortaleciendo los espacios multiactorales [...]" (REBÓN; SALSE, 2003, p. 6).

Desde la normativa del programa, se plantea entonces un enfoque que intenta "transformar las necesidades en oportunidades de acción colectiva" (MDS, 2006), a través del otorgamiento de créditos o subsidios para la producción en emprendimientos asociativos.

Por su parte, el Programa Trabajo Autogestionado, puesto en marcha a través del MTESS a fin del año 2003, se propone "contribuir a la generación de nuevas fuentes de trabajo y/o al mantenimiento de puestos existentes, a través de la promoción y el fortalecimiento de unidades productivas autogestionadas por los trabajadores" (ARGENTINA, 2004b). La fundamentación del programa alude a "la crisis socioeconómica sufrida en años anteriores" como originaria del "fenómeno de las empresas recuperadas"7, entendidas como una "iniciativa adoptada por los trabajadores para mantener o recuperar sus puestos de trabajo". A fin de promover la sustentabilidad y el desarrollo de estas experiencias, el Ministerio de Trabajo impulsa estrategias y acciones de apoyo técnico, económico y financiero. Asimismo, prevé la cofinanciación de capacitación y asistencia técnica y tiene como requisito el carácter asociativo de los proyectos, definidos como un modelo que:

[...] tienda a combinar la autonomía en la gestión con la propiedad social de los bienes/medios de producción; la orientación hacia la consecución de prácticas compatibles con el concepto de "trabajo de calidad", favoreciendo el trabajo productivo, la protección social y la equidad; el sustento solidario 
del emprendimiento en relación con sus miembros y la comunidad (ARGENTINA, 2004a).

Si examinamos los objetivos de los programas, podemos observar que si bien ambos intentan facilitar la inserción laboral de sus destinatarios, el Plan "Manos a la Obra" plantea como propósito explícito promover la "inclusión social" de los sujetos, a través de la generación de empleo. Los destinatarios de este plan son personas o grupos definidos como sujetos en "situación de vulnerabilidad", "de pobreza" o "de bajos recursos". Esta diferencia en la enunciación de los objetivos, supone la definición de los destinatarios de la política social como "excluidos" del mercado de trabajo y del sistema social, por ello, resulta necesario promover su "inclusión"» (HOPP, 2009). Además, en los lineamientos del Plan puede observarse, por un lado, una cierta idealización de la pobreza que da por supuesto que los destinatarios están dispuestos a asociarse con otros y así fortalecer los procesos de desarrollo local, ignorando la fragmentación social y las desiguales condiciones y recursos que presenta cada localidad o comunidad para afrontar los procesos de desarrollo. También, supone condiciones igualitarias de participación en los espacios de articulación multiactoral, como los consejos consultivos ${ }^{9}$, creados para elaborar los planes de desarrollo. Consideramos que no existe una "propensión natural" hacia ninguna práctica; además, es probable que las distintas experiencias, capacidades, recursos y grados de organización de los actores, determinen posibilidades diferenciales de participación e imposición de sus propuestas en estos espacios. La igualación de las relaciones y la posibilidad de "tomar parte" en la participación, entendida como la capacidad de incidir en la toma de decisiones (ROBIROSA, 1990) es una condición para construir (y las experiencias participativas que promueve el programa podrían estar dirigidas a esa construcción), pero no puede ser tomada como un punto de partida dado.

A diferencia del Plan "Manos a la Obra", el Programa Trabajo Autogestionado tiene como objetivo promover la inserción laboral o resguardar el empleo, y está destinado a:

Empresas/Fábricas recuperadas por los trabajadores, independientemente de la figura jurídica que adopten [...], Cooperativas de trabajo y/o de producción [...] y entidades con personería jurídica que nucleen cooperativas, empresas recuperadas o microempresas, para el desarrollo de procesos asociativos de producción, comercia-lización y fortalecimiento de cadenas de valor (ARGENTINA. 2004a).

La existencia de un programa destinado específicamente a estas unidades productivas tiene que ver con la conformación de un actor social y político - el Movimiento de Empresas Recuperadas, representado por distintas organizaciones $y$ federaciones, $\mathrm{y}$, actualmente, por la reciente creación de la Confederación Nacional de Cooperativas de Trabajo $^{10}$ (CNCT) que representa y lucha por conseguir el apoyo estatal para este tipo de experiencias, intenta institucionalizar una nueva forma de organización laboral y un nuevo sentido del trabajo. Las luchas simbólicas por la nominación se pueden dar de dos maneras:

En el aspecto objetivo, se puede actuar por acciones de representaciones, individuales o colectivas, destinadas a hacer ver y hacer valer ciertas realidades. [...]. Por el lado subjetivo se puede actuar tratando de cambiar las categorías de percepción del mundo y de la apreciación del mundo social (BOURDIEU, 1988, p. 137).

En este sentido, se puede observar desde el aspecto objetivo, el reconocimiento logrado y la visibilización de la realidad de las empresas recuperadas y cooperativas, expresadas en la creación de una línea de apoyo del MTESS, destinada exclusivamente a unidades productivas autogestionadas. En el aspecto subjetivo, se observa la disputa por una definición de trabajo diferente al empleo mercantil. La transformación de categorías a partir de las cuales se interpreta el mundo social "son la apuesta por excelencia de la lucha política” (BOURDIEU, 1988, p. 137), la imposición de una nueva definición, la autogestión, es resultado de dicha disputa.

\section{Políticas de desarrollo socio-productivo: nuevos roles y sentidos de la asistencia}

La Huella es una cooperativa que se dedica a la confección de calzado y ropa de trabajo. Su actividad principal es la elaboración de zapatos de seguridad, que venden a diferentes clientes, a empresas u otras cooperativas, produciendo también para otras marcas. La cooperativa está integrada por 14 socios, de los cuales 4 son mujeres y 10 varones. El trabajo se organiza en tres tipos de tareas: administración, producción y gestión, que son llevadas a cabo por distintos socios. Las tareas de gestión incluyen la articulación con otros actores gubernamentales y no gubernamentales, vinculados con la economía social y la búsqueda de recursos estatales y privados para fortalecer la actividad productiva. Si bien en la producción están ocupados la mayor parte de los integrantes, las actividades de articulación con otros y la gestión de recursos estatales, son fundamentales para el sostenimiento de la unidad productiva. Además, estas tareas demandan tiempo y esfuerzo y a diferencia de lo que sucede en una em- 
presa gestionada por un capitalista, son llevadas a cabo por los mismos trabajadores.

Desde su conformación, en el año 2006, esta cooperativa recibió dos subsidios para la compra de maquinarias, uno de ellos proveniente del Plan "Manos a la Obra" y el otro del Programa de Trabajo Autogestionado. También, proveyó de 800 pares de zapatos a un municipio de la Provincia de Buenos Aires, destinados a los integrantes de Cooperativas generadas a partir del Plan "Argentina Trabaja"11.

Aquí observamos una transformación del rol del Estado y de la política social, tradicionalmente vinculada con acciones asistenciales destinadas a individuos o familias. En el caso de los programas de desarrollo socio-productivo, la política social adquiere un nuevo rol vinculado a la promoción de formas de trabajo autogestionadas. Además, las unidades productivas, que son apoyadas por estos planes, no son sólo receptoras de los recursos otorgados por dichas políticas, sino que pueden ser proveedoras del Estado, situación que, por un lado, facilita la comercialización de la producción de la cooperativa y, por otro, habilita nuevas formas de relación entre Estado y sociedad, valoradas positivamente por los destinatarios de estas políticas.

Desde la mirada de los sujetos que conforman esta cooperativa, las políticas de desarrollo socioproductivo, son consideradas un recurso fundamental para fortalecer su actividad económica y su autonomía como trabajadores. Ante la pregunta acerca de la diferencia entre los planes asistenciales y los de desarrollo socio-productivo, los integrantes de la cooperativa dijeron lo siguiente:

Damián explica que las máquinas (que consiguieron a través del Plan 'Manos a la Obra' y del Programa Trabajo Autogestionado) son una ayuda. Sonia dice que para ella estos planes significan una mejora, porque te ayudan a comprar la máquina para trabajar. Pedro asiente y plantea que para él también es mejor, porque con 'otros planes te dan mercadería' o te alcanza para comprar comida, 'pero vos necesitás ropa, tenés otras necesidades, muchas necesidades' [...] Sonia le contesta: 'Los planes [se refiere al Plan Jefas y Jefes de Hogar Desocupados ${ }^{12}$ y otros con contraprestación laboral que se otorgaron durante la crisis del 2001] fueron un paliativo para la situación de ese momento, ahora, las máquinas te sirven para generar tus propios recursos' (Registro de Campo, 10 oct. 2009).

Los testimonios de los integrantes de esta cooperativa, que durante la crisis del 2001-2002 recibieron planes con contraprestación laboral y actualmente son socios de una cooperativa de trabajo, dan cuenta de una transformación del sentido que adquiere la asistencia en la vida de estos sujetos. A diferencia de la representación dominante acerca de los planes con contraprestación laboral y la discusión en torno al sentido de dicha contraprestación y si ésta era o no equivalente al trabajo ${ }^{13}$, los destinatarios de programas de desarrollo socio-productivo que han logrado poner en marcha el emprendimiento, no ponen en duda que lo que hacen es trabajar, aunque hayan recibido un subsidio. El sujeto de la asistencia ya no es el individuo, sino el grupo asociado o la unidad productiva autogestionada y esta situación permite construir una identidad más valorada, evitando así el estigma de la asistencia y habilitando nuevas relaciones comerciales y de cooperación, con las instituciones estatales.

\section{La implementación de las políticas de desar- rollo socio-productivo: "viejas" dificultades}

A pesar de los aspectos positivos y de la potencialidad para la generación de nuevas relaciones entre las instituciones estatales y las organizaciones de la sociedad civil, que promueven las políticas de desarrollo socio-productivo, en el proceso de implementación, observamos algunas dificultades que obstaculizan el desarrollo de los emprendimientos que éstas intentan impulsar.

Una de las socias de la cooperativa en la que realizamos el trabajo de campo, cuenta su experiencia de acceso al Plan "Manos a la Obra":

'Nosotros tuvimos que hacer movilizaciones durante dos años para conseguir las máquinas. Hicimos un proyecto que incorporaba 10 personas'. Finalmente, cuando recibieron el subsidio se dieron cuenta que en el Ministerio de Desarrollo Social 'recortaron el proyecto y el presupuesto, y nos dieron sólo 11 mil pesos'. Explica que con ese dinero no pudieron comprar todas las máquinas que habían propuesto, entonces, de las 10 integrantes que estaban en el proyecto original, sólo algunas podían trabajar, porque como no había suficientes máquinas, otras no tenían nada que hacer. 'Nos dieron las máquinas que quisieron y no fue coherente', dice. Luego, cuenta que también fueron a inspeccionar, a ver si estaban usando las máquinas, pero que a los emprendimientos que no estaban trabajando, no les dijeron nada. 'En el barrio, otros vendieron las máquinas y nadie les dijo nada' (Registro de Campo, 15 sept. 2009).

Como el emprendimiento textil en el que participaba no funcionó, Sonia se llevó las máquinas que había conseguido a través del plan y se sumó a la cooperativa en la que actualmente trabaja para poder usarlas colectivamente, porque como afirma ella, 'para eso nos las dio el Gobierno' (Registro de Campo, 31 jul. 2009). 
El relato de Sonia muestra las dificultades con las que se encontró para acceder al subsidio del Plan "Manos a la Obra" y poner en marcha el emprendimiento. Primero, se observa la lucha de la organización social en la que estaba para conseguir el acceso a los planes, a pesar del objetivo explícito del programa de promover la participación y fortalecer a los actores de la economía social. Cuando lograron el acceso al subsidio, éste no pudo garantizar la creación de fuentes de trabajo, lo cual debilitó al grupo y terminó por disolverse el emprendimiento. El "recorte del presupuesto" que denuncia Sonia nos lleva a reflexionar acerca del criterio utilizado para otorgar los financiamientos estatales. Parece ser que se financia "una parte", más allá de lo que el solicitante haya calculado y justificado ${ }^{14}$, según los recursos disponibles que haya para distribuir. Con lo cual, efectivamente, los proyectos se debilitan en muchos casos o se disuelven, como en la experiencia que relata Sonia. Finalmente, la posibilidad que ella encontró de integrarse a una cooperativa que ya estaba en funcionamiento y sumar las máquinas que había conseguido, permitió concretar la experiencia de trabajo asociado que buscaba y que, de algún modo, se sentía en la obligación de concretar, por el hecho de haberlas recibido por un plan del Estado. Sin embargo, no tuvieron la misma suerte las otras 9 emprendedoras que participaron en la lucha de la organización por conseguir el acceso al plan y en la elaboración del proyecto, pero se desanimaron frente a las dificultades para poner en marcha la actividad productiva.

Durante el desarrollo del trabajo de campo, los integrantes de la cooperativa se enteraron que les "salió el subsidio" del Programa Trabajo Autogestionado para la compra de una nueva máquina de corte que necesitaban, porque la que tenían era muy vieja, se rompía recurrentemente, lo cual atrasaba la producción, además, cuando estaba funcionando hacía muchísimo ruido.

Para poder recibir la máquina, que sería comprada por el MTESS y luego entregada a la cooperativa, acompañé al presidente de La Huella a firmar el convenio solicitado para efectivizar el subsidio. Como habían pasado varios meses desde la presentación del proyecto, el precio de la máquina había aumentado y con el monto asignado, no les alcanzaba para comprarla. Después de algunas gestiones ante el Ministerio y la averiguación de otros precios, pudieron resolver el problema y pasados cinco meses recibieron la máquina. El largo tiempo que demora el proceso de presentación de la propuesta, evaluación, hasta la efectiva asignación y entrega de los recursos otorgados por estos programas, es una de las principales dificultades del proceso de implementación de estas políticas (SIGEN, 2006, 2007; CORAGGIO; FEDERICO, 2006; MDS, 2007). Esta situación, tal como muestra la experiencia de la cooperativa, puede poner en riesgo la eficacia del recurso, en este caso, debido al aumento de los precios que dificultó la compra de la máquina solicitada ${ }^{15}$. A pesar de ello, el trabajo conjunto entre la cooperativa y los empleados del ministerio permitió realizar la compra y la renovación de esa máquina generó una mejora notable en las condiciones laborales de la cooperativa. Por un lado, agilizó la producción, ya que la nueva cortadora funcionaba automáticamente, con mayor velocidad y no necesitaba reparaciones. Por otro, disminuyó considerablemente el ruido que había en la sección de corte, lo cual mejoró mucho el ambiente de trabajo.

En el mes de julio del 2009, en el marco del trabajo de campo, ayudé a los integrantes de la cooperativa a gestionar un subsidio destinado a la capacitación en oficios. La solicitud se realizó en conjunto con otra organización que nuclea microemprendimientos textiles y que no tiene personería jurídica. Después de más de un año, aún no obtuvieron respuesta.

Desde el momento en que la cooperativa decidió presentar el subsidio, hasta que efectivamente pudo entregar el proyecto, pasaron más de tres meses. Las dificultades que tuvieron para armar la propuesta fueron muchas, algunas ligadas al diseño, la formulación del proyecto y el llenado de los formularios estandarizados del programa; otras relacionadas con los requisitos formales vinculados a la personería jurídica de la cooperativa y a la organización. Las políticas de desarrollo socio-productivo y las formas de autogestión del trabajo que éstas promueven, suponen un rol más activo de los sujetos, en el cual ellos mismos son los encargados de planificar, justificar y llevar adelante su propuesta.

Los problemas que tuvo la cooperativa revelaron el "choque" entre la lógica del funcionamiento estatal, los requisitos formales que éste exige y los tiempos burocráticos para la gestión de recursos. También, las condiciones en que desarrollan sus actividades en este tipo de emprendimientos, sus necesidades más inmediatas o "urgentes" y la experiencia de los socios de la cooperativa. Por un lado, las prácticas cotidia- 
nas de los participantes de estas organizaciones de la sociedad civil, generalmente, no están vinculadas con el tipo de tareas que requiere la presentación de un proyecto de este tipo, para plasmar por escrito los objetivos, planificar y calcular los resultados esperados de la producción a partir de los recursos solicitados al programa, etc. ${ }^{16}$. Por otro, el funcionamiento del Estado y la posibilidad de comunicar y evaluar las políticas estatales requiere, entre otras cosas, del registro de las acciones realizadas y de los proyectos financiados en complejos formularios, sumado al requisito de contar con personería jurídica para recibir dinero proveniente del subsidio.

Por otra parte, en el proceso de presentación de proyectos socio-productivos, es fundamental la capacitación de los agentes estatales, a fin de que puedan asesorar a los destinatarios para la formulación de un proyecto viable ${ }^{17}$, acorde con las capacidades y recursos de los destinatarios y de la localidad en la que se encuentran. En este sentido, es necesario considerar los condicionamientos que imponen las particularidades y experiencias previas de implementación de programas sociales en los distintos Municipios (MERLINSKY; ROFMAN, 2004). Es así que los técnicos municipales, provenientes de áreas de acción social cuyas prácticas se vincularon históricamente con la distribución de recursos asistenciales, los cuales intervienen actualmente en el Plan "Manos a la Obra" cumpliendo un nuevo rol de promoción y asistencia para los emprendimientos socio-productivos, no cuentan con la capacitación necesaria para realizar dichas tareas.

\section{Reflexiones finales}

Las políticas de promoción de desarrollo socioproductivo analizadas nos invitan a revisar los postulados de la sociología política moderna que

[...] ha sido fijada definitivamente por la creencia empírica de que había 'sólo dos esenciales' encumbrados protagonistas de la organización social que forjaron el mundo moderno: 'el moderno Estado administrativo y la economía de mercado' (SOMERS, 1996, p. 256).

Esta lectura se expresó en la dicotomía público-privado que caracteriza al pensamiento moderno. Las cooperativas o emprendimientos promovidos por las políticas forman parte de un tercer espacio, en el que se desarrolla la vida pública participativa que no responde ni al individualismo del mercado, ni a la lógica del Estado. Pero que tampoco se asocia a la idea de una sociedad civil prepolítica o despolitizada.

El diseño de las políticas de desarrollo socioproductivo estudiadas y las nuevas relaciones entre
Estado y sociedad que éstas promueven cuestionan la idea de un Estado "exterior" a la sociedad vinculada con la representación tradicional de la política, entendida como un ámbito institucional restringido y claramente delimitado. Representación según la cual "eran prácticas políticas (las) que se referían al gobierno, al parlamento, a los partidos y en términos generales al Estado" (LECHNER, 1984, p. 127). Estas políticas problematizan la representación del Estado devenida del sentido común, que "presenta a éste como una organización burocrática que concentra el ejercicio del poder sobre la sociedad" (GRASSI, 2003, p. 107). El diseño y los objetivos de estos programas suponen, en el plano normativo, y requieren para su implementación el protagonismo de sus destinatarios, de las organizaciones sociales y de los distintos niveles gubernamentales. Esta participación es central en la construcción de las estrategias de desarrollo local y para el sostenimiento de las unidades productivas en el largo plazo. Por ello, consideramos que es necesario revisar el supuesto de igualdad de condiciones de participación que subyace a estos programas, a fin de alcanzar, a través de la implementación de estas políticas sociales, la efectiva posibilidad de tomar parte en los procesos de articulación Estado-sociedad civil que estos programas promueven.

En cuanto al Estado, estos planes redefinen su rol y proponen a éste como promotor de nuevas formas de trabajo autogestionadas. La incipiente institucionalización de las políticas socio-productivas, muestra una redefinición de las causas y los ámbitos de responsabilidad del problema del desempleo, abriendo el juego para pensar nuevas estrategias de abordaje del mismo y poniendo la responsabilidad de su resolución tanto en los grupos de trabajadores autogestionados, como en las intervenciones estatales para promover y fortalecer estas experiencias. Además, se habilitan nuevas formas de relación entre Estado y sociedad civil, que cuestionan la idea del sujeto como receptor pasivo de la asistencia.

$\mathrm{Si}$ bien podemos afirmar que estas políticas sociales son novedosas, por su enfoque y metodología, este artículo mostró algunos de los obstáculos que se presentan en el proceso de implementación de las mismas. Estos problemas dificultan el desarrollo y la sostenibilidad de los emprendimientos en el largo plazo. Así, una de las limitaciones centrales que observamos es que las políticas de promoción del desarrollo socio-productivo, se despliegan al margen de un marco legal adecuado y una regulación eficiente de las condiciones de producción y comercialización, de protección y de seguridad de los productores. Sumado a la contradicción que plantea el impulso del desarrollo socioeconómico, a través de políticas focalizadas e implementadas en su mayoría por el Ministerio de Desarrollo Social, cuyas 
intervenciones estuvieron históricamente vinculadas con la asistencia ${ }^{18}$.

Por último, consideramos que es necesario profundizar el análisis de estas políticas, a fin de fortalecer la construcción de un sector de la economía social basado en relaciones sociales más igualitarias, formas de producción económica centradas en el trabajo, orientadas a la reproducción ampliada de la vida de todos y que no estén subordinadas al capital.

\section{Referencias}

ABRAMOVICH, A. Emprendimientos productivos de la economía social en Argentina: funcionamiento y potencialidades. In: CIMADAMORE, A. La economía política de la pobreza. Buenos Aires: CLACSO, 2008, p. 221-259.

ARGENTINA. Resolución MDS n. 1375/2004. Buenos Aires, 13 abr. 2004.

Resolución MTESS n. 194/2004, por la que se reglamenta el Programa Trabajo Autogestionado. Buenos Aires, 21 abr. 2004a.

Resolución MDS n. 3182/2009. Programa de Ingreso Social con Trabajo-Argentina Trabaja. Buenos Aires, 6 ago. 2009.

BOURDIEU, P. Cosas dichas. Buenos Aires: Gedisa, 1988.

CNCT-Confederacion Nacional de Cooperativas de Trabajo. Revista Autogestión Argentina, n. 5, Buenos Aires: CNCT, 2009.

CORAGGIO, J. L.; FEDERICO, A. Emprendimientos socioeconómicos asociativos: su vulnerabilidad y sostenibilidad. Informe final. Buenos Aires: Secretaria de Políticas Sociales y Desarrollo Humano, MDS-ICO/UNGS, 2006.

DANANI, C. La política social como campo de estudio y la noción de población-objeto. In: HINTZE, S. Politicas Sociales. Contribuciones al debate teórico-metodológico. Buenos Aires: Eudeba-CEA, 1996, p. 21-38.

DINATALE, M. El festival de la pobreza: el uso político de planes sociales en la Argentina. Buenos Aires: La Crujía, 2004.

GEERTZ, C. Conocimiento local. Buenos Aires: Paidós, 1994.

GRASSI, E. Políticas y problemas sociales en la sociedad neoliberal. La otra década infame. Buenos Aires: Espacio, 2003.
Integración y necesidades sociales. Reflexiones desde el punto de vista de la igualdad. In: TERCER CONGRESO ARGENTINO DE POLÍTICA SOCIAL, 18 al 20 oct. Anais... Buenos Aires, 2006.

HELLER, A. Sociología de la vida cotidiana. Barcelona: Ediciones Península, 2002.

HOPP, M. La construcción de nuevos sentidos del trabajo en las políticas de promoción de desarrollo socioproductivo en la Argentina post-convertibilidad. Revista Margen de Trabajo Social, Buenos Aires: Margen Editorial, n. 55, p. 1-13, 2009.

Planes sociales, contraprestación y huidas de la asistencia. In: GRASSI, E.; DANANI, C. El mundo del trabajo y los caminos de la vida, trabajar para vivir, vivir para trabajar. Buenos Aires: Espacio, 2009a, p. 263-296.

Políticas de promoción de desarrollo socioproductivo en la Argentina post-convertibilidad: condiciones de la integración social y económica. Tesis (Magister en Políticas Sociales) - Facultad de Ciencias Sociales, Universidad de Buenos Aires, 2010.

LECHNER, N. Especificando la política. In: VEGA, J. Teoría y política de América Latina. México: Libros del CIDE, 1984, p. 127-143.

MDS-Ministerio de Desarrollo Social. Lineamientos de políticas sociales. 2006. Cuaderno 1. Buenos Aires: Secretaría de Políticas Sociales y Desarrollo Humano, 2006.

Evaluación de medio término. Plan Nacional Manos a la Obra. Estudio cualitativo. Análisis de casos, 2007. Buenos Aires: Secretaría de Políticas Sociales y Desarrollo Humano-PNUD, 2007.

La construcción pública del desarrollo local. La experiencia del Plan Nacional Manos a la Obra, 2006. Buenos Aires: Secretaría de Políticas Sociales y Desarrollo Humano-PNUD, 2007a.

MERLINSKY, G.; ROFMAN, A. Los programas de promoción de la Economía Social: ¿una nueva agenda para las políticas sociales? In: FORNI, F. Caminos solidarios de la economía argentina. Redes innovadoras para la integración. Buenos Aires: Ciccus, 2004, p. 161-190.

PORTANTIERO, J. C. El tiempo de la política. Construcción de mayorías en la evolución de la democracia argentina 19832000. Buenos Aires: Temas Grupo Editorial, 2000.

REBÓN, M.; SALSE, G. Plan Manos a la Obra: dificultades y desafíos de su gestión. In: FORO FEDERAL DE INVESTIGADORES Y DOCENTES, 2007, 27 agosto. Buenos Aires, 2007. 
ROBIROSA, M. Turbulencia y planificación social, lineamientos metodológicos de gestión de proyectos sociales desde el Estado. Buenos Aires: Siglo XXI, 1990.

RUGGIERI, A.; MARTÍNEZ, C.; TRINCHERO, H. Las empresas recuperadas en la Argentina: Informe del segundo relevamiento del Programa Facultad Abierta. Buenos Aires: Seube-UBA, 2005.

SIGEN-Sindicatura General de la Nación. Informe de Auditoría n. 1 e n. 2, 2006. Disponible en: 〈http:// www.sigen.gov.ar/informes.asp >. Acceso en: 6 oct. 2009.

Informe de Auditoría n. 1, 2007. Disponible en: <http://www.sigen.gov.ar/informes.asp $>$. Acceso en: 6 oct. 2009.

SOMERS, M. Narrando y naturalizando la sociedad civil y la teoría de la ciudadanía: el lugar de la cultura política y de la esfera pública. Revista Zona Abierta, Madrid: Editorial Pablo Iglesias, n. 77/78, p. 255-337, 1996/97.

\section{Notas}

1 Discurso pronunciado por Carlos Tomada, Ministro de Trabajo, Empleo y Seguridad Social, en el marco de La presentación oficial del Seguro de Capacitación y Empleo. Buenos Aires, 13 de Febrero de 2006.

2 Disponible en: <http://www.trabajo.gob.ar/planesy programas/masymejor.asp >. Acceso en: 20 jun. 2009.

3 En el marco del Plan de Promoción del Empleo, el MTESS implementa el Programa Herramientas por Trabajo y el Programa de Inserción Laboral-línea autoempleo, ambos destinados a los trabajadores desocupados con el propósito de facilitar su reinserción, a través de la realización de emprendimientos productivos individuales o asociativos y del Programa de Trabajo Autogestionado, destinado a las empresas recuperadas por sus trabajadores y cooperativas autogestionadas.

4 Definimos a la sociedad civil "como una esfera organizada de la vida social en la que actores colectivos expresan intereses, valores y efectúan demandas al Estado". Desde esta perspectiva no idealizamos las organizaciones de la sociedad civil, pensadas como entidades más eficientes que podrían reemplazar la ineficacia estatal, sino como actores con intereses, prácticas, valores y discursos diversos, insertas en contextos determinados (PORTANTIERO, 2000).

5 Tomaremos las normativas del Plan Nacional de Desarrollo Local y Economía Social "Manos a la Obra” y del Programa Trabajo Autogestionado, debido a que son los programas de desarrollo socio-productivo en donde se observa más claramente esta nueva división del trabajo y la articulación entre Estado y Sociedad Civil. Por otra parte, la cooperativa en la que realizamos el trabajo de campo recibió apoyo de estos dos programas.

6 Para reconstruir el diagnóstico a partir del cual se diseñó el Plan "Manos a la Obra" nos basamos en MDS (2006, 2007, 2007a).

7 Entendemos por empresa recuperadaa “todas aquellas firmas, que, habiendo detenido la producción y transitado una cesación de pagos con sus acreedores (ya sea porque se encontraba en concurso preventivo sin una resolución que garantice la continuidad de la empresa o porque directamente se presentó en quiebra) fueron reabiertas, a partir de la iniciativa de los trabajadores para sostener la fuente de trabajo, reiniciando las actividades productivas con la intervención directa de los mismos en la administración" (RUGGIERI; MARTÍNEZ; TRINCHERO, 2005, p. 23). Las empresas recuperadas, son un fenómeno social y económico que surge como consecuencia del deterioro de la sociedad salarial, en un contexto de desempleo estructural producto de políticas neoliberales. La experiencia de empresas recuperadas, pone en cuestión la idea del desempleado como "excluido" y destinatario de políticas sociales asistenciales. En este sentido, la consigna de las empresas recuperadas ha sido desde un principio "Ocupar, Resistir y Producir”.

8 El término más utilizado en las normativas y documentos del Ministerio de Desarrollo Social y el Ministerio de Trabajo es "inclusión social", aunque también en algunos pasajes y utilizado como sinónimo de inclusión se refieren a la integración social. Aunque no podemos hacer un desarrollo de esta cuestión, queremos señalar que existen diferencias teóricas entre ambos conceptos y que es necesario tener en cuenta para el análisis de las políticas sociales. Al respecto, remito al lector a Grassi (2006) y Hopp (2010), en donde se analizan estas diferencias.

9 Los consejos consultivos se crearon en el año 2002, a partir de la implementación del Plan Jefas y Jefes de Hogar Desocupados, con el objetivo de administración y control de los subsidios que otorgaba el programa. Al respecto, ver Hopp, (2009a). Actualmente, éstos continúan participando en la ejecución de distintos Programas Sociales. Los consejos consultivos son una institución novedosa, en la que participan distintos actores sociales y políticos que permiten a los destinatarios de los programas y a las organizaciones de la sociedad civil participar en la implementación, el control y en algunos casos en el diseño de la política social.

10 La Confederación está constituida por Fecootra, Facta, Fecooaport, Fecootra-Un Florencia Varela, Fecootra-Un Corrientes, ANTA, Fecotrar, Ferycootra, Padre Carlos Mugica, Federación Evita, Reconquista, Fecosol, FECOSanta Cruz, Cotrafer, Adiccra, Ificotra, Federación Obra Padre Mugica, Umiscoop, Federarty Red Gráfica(CNCT, 2009).

11 Como mencionamos en la introducción, el Plan "Argentina Trabaja" comenzó a implementarse en a gosto del 2009, con 
el propósito de generar trabajo, a través de la creación de cooperativas, que se dediquen a realizar tareas vinculadas, principalmente, con la obra pública y el mejoramiento barrial. Considerando el alcance previsto (100 mil puestos de trabajo) y la magnitud del presupuesto destinado (1500 millones de pesos, que equivalen aproximadamente a 375 millones de dólares), "Argentina Trabaja" puede considerarse el principal programa socio-productivo del período 2003-2010.

12 Este plan fue creado en enero del 2002 y puesto en marcha en mayo de ese mismo año para hacer frente de manera urgente a las consecuencias de un proceso que comenzó en la década del 90 y culminó en el estallido de la crisis en diciembre del 2001, llegando a tener, a dos meses de su lanzamiento, casi dos millones de destinatarios y un presupuesto de 3.500 millones de pesos (DINATALE, 2004). El Plan Jefas y Jefes de Hogar Desocupados estuvo destinado a jefas y jefes de hogar en situación de desempleo que tuvieran hijos menores de edad o discapacitados a cargo. El Plan otorgaba un subsidio de 150 pesos mensuales (aproximadamente 50 dólares en ese entonces) y exigía la realización de una contraprestación laboral o capacitación de 20 horas semanales.

13 Al respecto, ver Hopp (2009a).

14 La solicitud de financiamiento de un proyecto productivo ante el Plan "Manos a la Obra" requiere la explicitación detallada de los objetivos, métodos y recursos necesarios para llevar adelante el emprendimiento. Además, se deben acompañar 3 presupuestos que indiquen el valor y el tipo de recursos materiales necesarios.

15 También pueden darse otro tipo de situaciones que restan eficacia a los recursos otorgados, por ejemplo, en el caso de subsidios destinados a la compra de insumos para actividades estacionales, el tiempo de espera puede implicar que éstos se vuelvan inadecuados. Otro problema es el desaliento generado por la espera y la consiguiente disolución del grupo asociado, en los casos en que éstos no estuvieran en funcionamiento antes de pedir el subsidio.

16 En este sentido, es elocuente el comentario de un cooperativista que explica que la Organización en la que participa "nos dio una mano con la contabilidad y con los papeleos, de eso nosotros no sabemos nada, nosotros solo sabemos trabajar" (Registro de Campo, 19 dic. 2009).

17 Diversas auditorías del Plan Nacional de Desarrollo Local y Economía Social "Manos a la Obra" muestran las dificultades de los emprendedores para el desarrollo y sostenibilidad de los proyectos socio-productivos (SIGEN, 2006, 2007).

18 El Plan "Manos a la Obra" tiene un alcance mucho mayor que el Programa Trabajo Autogestionado. El primero asistió, entre los años 2003 y 2006, a 62.569 unidades productivas, con un total de 534.115 integrantes (MDS, 2007). El segundo, entre los años 2003 y 2008, brindó asistencia técnica a cerca de 100 empresas y fábricas recuperadas por sus trabajadores para el fortalecimiento de sus unidades productivas y otorgó subsidios a 139 empresas autogestionadas (ARGENTINA, 2009).

\section{Malena Victoria Hopp}

malenahopp@yahoo.com.ar Magister en Políticas Sociales de la Universidade de Buenos Aires (UBA)

Profesora en la Facultad de Ciencias Sociales, UBA Becaria doctoral del Consejo Nacional de Investigaciones Científicas y Técnicas de La República Argentina (Conicet), Instituto de Investigaciones Gino Germani, Facultad de Ciencias Sociales (FSOCUBA)

Direción: Prof. Dra. Estela Grassi

\section{FSOC - UBA}

Instituto de Investigaciones Gino Germani Facultad de Ciencias Sociales

Pte. J. E. Uriburu $9506^{\circ}$.

1114

Buenos Aires - Argentina 